\title{
Prácticas docentes que promueven el compromiso social de sus alumnos
}

\section{Teaching Practices to Promote Social Commitment on Students}

\author{
Juan Carlos Silas Casillas
}

Instituto Tecnológico y de Estudios Superiores de Occidente (iteso), MéXico

silasjc@iteso.mx

Laura Angélica Bárcenas Pozos

Universidad Iberoamericana Puebla, México

laura.barcenas@iberopuebla.mx

https://doi.org/10.48102/didac.2021..78_JUL-DIC.79

8

\section{RESUMEN}

Este artículo describe las prácticas que realizan los profesores universitarios interesados en ir más allá de lo técnico-disciplinal para propiciar el compromiso social de sus alumnos. Aquí se reportan los hallazgos de una investigación realizada durante 2017 y 2018 en seis instituciones del Sistema Universitario Jesuita. Los dos tipos de prácticas son discursivas y de acercamiento a la realidad.

Palabras clave: Compromiso social; prácticas docentes; universidad.

\section{ABstract}

This text seeks to illustrate the practices carried out by university professors in their interest to go beyond the technicalldisciplinary and promote the development of social commitment of their students. The findings of an investigation carried out during 2017 and 2018 in 6 institutions belonging to the Jesuit University System are reported. The two types of practices are discursive and approach reality.

Keywords: Social Commitment; Teaching Practices; University.

Fecha de recepción: 14/04/2021

Fecha de aceptación: 10/05/2021

\section{Introducción}

En las instituciones universitarias es conocida la trascendencia que tienen las prácticas de los profesores como los actores involucrados de forma más cercana en la transformación de los estudiantes. La literatura profesional reporta los beneficios de incluir asignaturas orientadas a promover el compromiso social (Torrego Egido \& Monjas Aguado, 2007; Cacho Sánchez \& Llano Martínez, 2014). Otros se centran en la acción directa de los profesores y resaltan que 
son los académicos quienes, a través de encarnar los valores adecuados, marcan la pauta a los estudiantes (López Zavala, 2007; Aguirre, De Pelekais \& Paz, 2012). Por otro lado, Porta y Flores (2017) enfatizan la trascendencia de la cercanía emocional de los profesores en relación con sus alumnos y señalan que los vínculos de "hospitalidad" posibilitan el compromiso de los estudiantes con los demás.

Martínez-Otero (2008) construyó una tipología, retomada por Suescun Guerrero (2015), que clasifica la intención del discurso del profesor y propuso cinco perfiles: 1) el enseñante, claramente instructivo; 2) el progenitor, interesado en la persona; 3) el presentador, un ameno expositor; 4) el político, con sesgo "propagandístico", y 5) el predicador, con una retórica moral. Los dos últimos se relacionan con los hallazgos de este proyecto, reportados a continuación.

Los datos obtenidos en el proyecto de investigación sobre las prácticas docentes que promueven el compromiso social de los estudiantes universitarios ofrecen pautas concordantes con la literatura profesional y aportan pistas para un mayor impacto en la formación integral de los alumnos. Los hallazgos reportados en este texto son un componente de un proyecto amplio que implicó la aplicación de un cuestionario a una muestra representativa de docentes en seis universidades y la conducción de 49 entrevistas con académicos sobre cómo entienden el compromiso social y qué han hecho para promoverlo entre sus pupilos.

Los profesores que fueron invitados a participar en la entrevista y a hacer explícitas sus prácticas se eligieron por tener un perfil alto en el instrumento cuantitativo (Cuestionario de Valores de Schwartz). Se preservó un balance en términos de antigüedad en la institución (menos de un año, entre uno y tres, entre tres y cinco, cinco a ocho, más de ocho) y área disciplinar del departamento de adscripción (económico-administrativas; ingeniería y ciencias exactas; ciencias políticas y derecho; psicología, educación y filosofía; nutrición y salud; ciencias sociales, comunicación y afines; y arte, arquitectura y diseño). Se observaron otras características personales, como edad, género, grado académico y licenciatura de formación inicial, pero sin que influyeran en la selección de los participantes, pues no se registró que representaran un sesgo en las prácticas.

Antes de describir las acciones es importante señalar que no se pretendió encontrar la frecuencia con que suceden, sino caracterizar las prácticas que los docentes consideran exitosas en la promoción del compromiso social. Fue evidente que las prácticas discursivas suceden con mayor frecuencia, pues requieren menor coordinación con otras instancias. Las acciones se pueden englobar en dos grupos: 1) las discursivas y 2) las de acercamiento a la realidad externa. En el primero, el medio fundamental es la expresión verbal y se da regularmente dentro del aula. El segundo incluye acciones que ponen al alumno en contacto directo con la desigualdad cotidiana.

El tipo de práctica empleada depende mayormente de la índole de la asignatura: cuando es más teórica, la actividad del docente es más discursiva; si es más práctica, se enfoca en intervenir o acercar a los alumnos a la realidad.

\section{Prácticas discursivas}

Las prácticas discursivas tienen lugar usualmente en la interacción maestro-alumno. El principal medio de transmisión de ideas es el discurso, la palabra hablada, a la manera señalada por Martínez-Otero (2008). Las modalidades más evidentes son:
a) Exposición y diálogo
b) Confrontación e ironía
c) Reflexión enfocada
d) Debate
e) Colaboración entre alumnos
f) Lectura de textos

Lo común en estas prácticas es la presentación convincente de ideas sobre las desigualdades sociales, acompañadas de preguntas detonadoras de diálogo sobre el tema visto y el compromiso social. Es usual que se hable del comportamiento adecuado de un profesional con compromiso social y mostrar ejemplos. Regularmente se concluye con un análisis de los

42 - Prácticas docentes que promueven el compromiso social de los alumnos Juan Carlos Silas Casillas, Laura Angélica Bárcenas Pozos. DIDAC 78 (2021): 41-48 
elementos de éste. Esto se produce de una forma muy cercana al concepto de triángulo de la interactividad de Coll y Solé (2002), en que interactúan el docente, los alumnos y el contenido en el contexto del aula (en este caso, el contenido es el compromiso social).

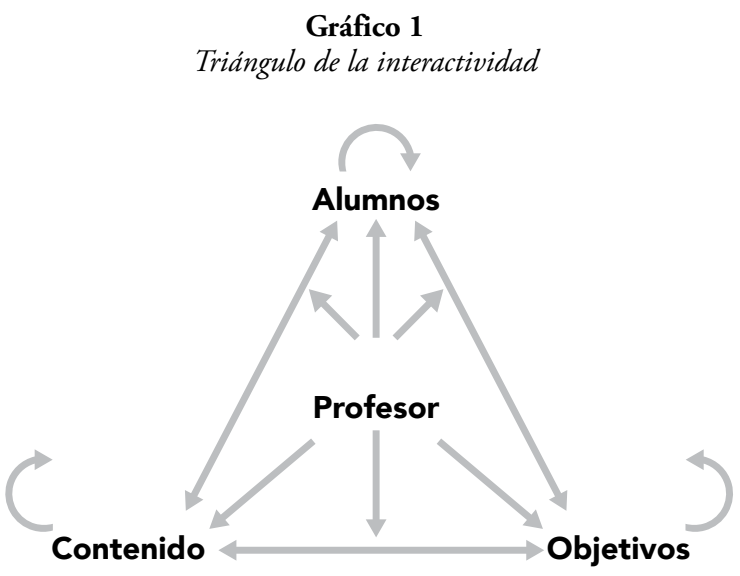

(Colly Solé, 2002)

Uno de los temas recurrentes es la responsabilidad. Los profesores entrevistados muestran su relevancia y aplicación, señalan ejemplos y comparten vivencias para hacer ver a sus alumnos la importancia de ser responsables como estudiantes, profesionales y ciudadanos. Por otro lado, es común que el docente se presente como un profesional que critica y cuestiona la manera individualista de trabajar en la sociedad actual, y busca que sus alumnos piensen en formas de aportar socialmente. En los próximos párrafos se transcriben testimonios de cada modalidad de prácticas discursivas.

\section{a) Exposición y diálogo}

Con frecuencia, los docentes comparten sus experiencias laborales o su formación para hablar sobre el compromiso social con sus alumnos:

Siempre en la primera clase les digo, ¿una empresa para qué sirve? Me contestan, no, para generar utilidades a los accionistas, lo del libro de texto; les digo: no, está para generar y agregar valor a los empleados, a los proveedores y al gobierno y a la ecología y a todos, ¿por qué? porque si no lo ven desde un punto de vista holístico, no lo ven desde un punto de vista integral, el balance se puede romper e incluso cuesta (Institución 1, profesor 37).

A través del discurso promueven no hacer lo que no es correcto, incluso cuando la mayoría lo hace:

Que los otros lo hagan no quiere decir que ustedes van a poner eso en práctica y mientras nosotros actuemos dentro de nuestra ética, vamos a hacer un buen trabajo, no hace falta caer en eso (Institución 2, profesor 1071).

También se aborda la idea de que se aprende para servir y no sólo para ganar dinero:

La frase sí entonces, yo siempre la he estado manejando con los chicos diciéndoles eso y haciéndoles ver que es un servicio. Los que se vienen a trabajar también lo viven, lo ven como yo trato a los clientes, cómo vemos las cosas, cómo resolvemos los proyectos, con una finalidad de servicio, no con una finalidad de querer cuánto me vas a pagar por este trabajo, ¿no? (Institución 1, profesor 65).

Los docentes usan su mensaje para que los alumnos vean la utilidad social de sus aprendizajes y los invitan a resolver problemas desde su profesión:

Escojan por lo menos uno; son demasiados, pero si escogen uno, se especializan en eso, tiene que ver con tu perfil, con tu carrera, pues ya basta con eso, porque si entre todos nos dividimos la tarea, pues todas esas tragedias que vemos en las noticias pueden tener una solución o al menos podemos avanzar a su solución (Institución 2, profesor 347).

Por medio del discurso, los docentes buscan concientizar a sus alumnos sobre cómo comportarse como dueños de empresas, considerando que algún día lo serán:

La otra son las nóminas, que sean justos; les digo, lo mejor que pueden hacer ustedes como jefes es 
ser justos, no agandallarse, no, por ahorrarse unos pesos bajando los costos, no pagando unos salarios justos, porque les digo, es que no se ponen a pensar lo valioso de la gente, cuando la gente es bien atendida, cuando es tratada con dignidad, la gente da más, rinde más que cuando los traen como perico a toallazos, les hacen chicanada y media (Institución 2, profesor 1126).

\section{b) Confrontación e ironía}

Otro tipo de acciones al interior del aula implican confrontar las ideas de los alumnos respecto a la sociedad. Esto puede ser a través de diferentes acciones, como cuestionar algunos conocimientos o formular preguntas. La formulación de preguntas está asociada con la ironía que pretende propiciar la reflexión sobre la desigualdad y la necesidad de asumir un compromiso social:

Sí, definitivamente siempre hago ese ejercicio ¿por qué?, porque les hago una pregunta: "Jóvenes, ¿ustedes están de acuerdo en que se les quite $15 \%$ de su salario?, sí, sí, a ver, ¿por qué sí?, ¿por qué no?". "Por esto y por esto." "A ver, tú no, ¿por qué?" "Por esto y esto." "Ah, te voy a poner un ejemplo drástico pero real, que pasa esto". (Institución 3, profesor 160).

\section{c) Reflexión enfocada}

En este tipo de prácticas los docentes aprovechan los temas de la clase para detonar reflexiones sobre el compromiso social, independientemente del tema que estén abordando.

Este tipo de temas desde luego que impactan al alumno, ayer vi sus caras de impacto y su disgusto, pero no puede quedar ahí; les pido, les exhorto a que piensen qué van a hacer, no se vale nada más estar leyendo el tema, platicándolo en el salón; no puede quedar ahí (Institución 2, profesor 38).

Hay profesores que quieren favorecer la reflexión sobre la responsabilidad de ser universitarios frente al resto de la población:
Al principio del curso yo no doy clase el primer día, platico con ellos, les digo quién soy, cómo soy [...]. Platico sobre la importancia de que sean y que busquen la forma de ser más cultos porque tenemos esa obligación como universitarios [...] en ese contexto les comento que los universitarios tenemos que leer, ¿por qué?, porque tenemos que ser más cultos, pero para qué queremos ser más cultos, para tener mayor capacidad de autocrítica y de crítica (Institución 2, profesor 1100).

\section{d) Debate}

Algunos docentes asumen que deben generar conciencia en sus estudiantes acerca de que estudiar una profesión implica ponerla al servicio de los otros:

Es muy importante que los alumnos tengan conciencia de esa gama que tienen en un momento dado, que la profesión puede y debe estar al alcance de toda la población (Institución 2, profesor 38$)$.

Otros buscan que sus alumnos reflexionen sobre las implicaciones de sus eventuales responsabilidades como patrones o empresarios:

Pues simple y sencillamente desde siendo honestos en cuestiones de "oye, vamos a vender este producto a un precio justo o vamos a pagar un salario justo o vamos a pagar impuestos de una manera justa”; todo eso pues realmente te va a $[$ sic $]$ haciendo la conciencia de... de ya de tu actividad laboral (Institución 3, profesor 136).

También hay profesores que plantean escenarios hipotéticos a sus alumnos y les preguntan qué harían en determinadas circunstancias:

Empiezas a crear, este, la conciencia o sea a despertarle su conciencia lo que es el bien y el mal y... y cómo contribuir o de plano entonces si estos del "sí, qué harías si..." (Institución 3, profesor 109).

44 - Prácticas docentes que promueven el compromiso social de los alumnos Juan Carlos Silas Casillas, Laura Angélica Bárcenas Pozos. DIDAC 78 (2021): 41-48 
El ejercicio del debate sobre aspectos controversiales con los estudiantes fue referido como productivo:

Y empieza el debate entonces les pongo se llama "qué harías si" esa clase particularmente, híjole, está como que muy ligada al compromiso social y lo que es la moral, lo que es los valores porque puedes tener muchas variaciones; puse muchas situaciones de ese tipo para que ellos entendieran (Institución 3, profesor 109).

e) Colaboración entre alumnos

Varios profesores señalaron la importancia del trabajo colaborativo entre alumnos como afianzador de relaciones, de la confianza interpersonal y como un medio para reconocer las ventajas de trabajar con otros. Dos testimonios notables son:

Mi idea pues, pues es buscar que haya colaboración entre ellos, que se entiendan, que platiquen, que se conozcan y que busquen, ahora sí, entre ellos mismos, su bien común, al trabajar juntos, para lograr ese resultado en esos proyectos o actividades, pues, juntos, que sea el resultado de lo que ambos hayan hecho o de acuerdo de como hayan decidido trabajar, entonces para mí ahí va esa parte de compromiso social (Institución 1, profesor 287).

Si tú estás trabajando en equipo es porque empiezas a confiar en los demás y empiezas a interactuar más y esa relación de confianza crea lazos que pueden ayudar a crear cosas más positivas. En, cambio, $[s i c]$ quienes quieren trabajar siempre solos es porque desconfían de los demás y quieren siempre ver su propio beneficio (Institución 1, profesor 737).

\section{f) Lectura de textos}

Varios académicos recurren a las lecturas pensando que sirven para que los estudiantes adquieran las habilidades de confrontar con la realidad, además de que obtienen herramientas para trabajar:
Antes no se usaba que leyeran teoría, ahora sí... sí los hacía leer. Una, porque tenían que confrontar la realidad y otra, porque no todos tenían las herramientas para trabajar (Institución 1, profesor 65).

Queda claro que detrás de las prácticas discursivas referidas hay cuatro intenciones fundamentales:

1) Sensibilizar a los alumnos sobre realidades diferentes y maneras distintas de proponer el bienestar en la comunidad.

2) Evidenciar la existencia de experiencias exitosas en este tipo de acciones.

3) Resaltar la utilidad de poner el conocimiento al servicio de otros.

4) Identificar situaciones de conflicto.

Estas intenciones no se manifiestan todo el tiempo, pero es claro que alguna tiene lugar en cada práctica discursiva. Pese a la trascendencia del mensaje verbal constante, resulta evidente que la toma de conciencia no se traduce automáticamente en acciones positivas para los menos favorecidos. Sin embargo, los testimonios recabados permiten concebir que hay un impacto en los alumnos. Es posible que el efecto sea temporal y que dure unos días, por lo que se requiere que este tipo de mensajes y prácticas se den de forma reiterada y concertada entre los académicos.

\section{Prácticas de acercamiento a la realidad externa}

Además de las prácticas discursivas, existen las que acercan a los alumnos a realidades de desigualdad social. Éstas se enfocan en conocer el fenómeno "de

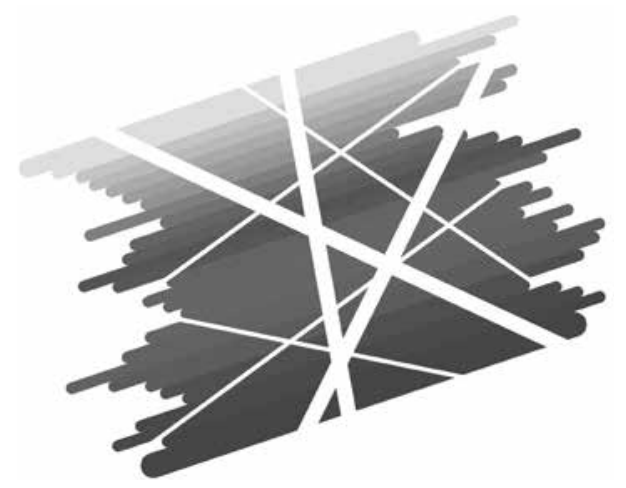


primera mano" y analizar cuáles son los actores y factores que influyen en él y las maneras en que un estudiante universitario puede actuar.

Las cuatro modalidades que se detectaron son:
a) Contacto directo
b) Aprendizaje situado
c) Trabajo localizado
d) Vivencia vicaria

A continuación, se describen y ejemplifican.

\section{a) Contacto directo}

El contacto directo es la práctica más potente en el trabajo realizado por los profesores para promover el compromiso social de los alumnos. Se trata de llevarlos a conocer y reconocer realidades distintas a las propias. Son acercamientos intencionales con "los otros" y la subsecuente reflexión personal y grupal. Una frase paradigmática sobre esto es la siguiente:

Lo que hicimos con los alumnos fue trabajar con los artesanos. Hicimos muchísimas visitas con los artesanos para trabajar con ellos y, sobre todo, para valorar mucho más sus técnicas, aunque nosotros no pudiéramos hacer nada, fuimos a todos los talleres de los artesanos. Son 26, pero en realidad fuimos como a 16 talleres. Realmente es su casa. (Institución 1, profesor 1080).

b) Aprendizaje situado

El aprendizaje situado requiere que "lo que será aprendido" tenga lugar en un entorno real y una práctica equivalente. Varios profesores dedican bastante tiempo de su asignatura a actividades de este tipo y piden a los alumnos que reflexionen al respecto.

El proceso de que el alumno empatice con el usuario es a lo que más le echamos trabajo. Por ejemplo, si son cuatro meses en [la asignatura] tenemos dos meses y medio de investigación. Para que vayan, conozcan el problema, hablen con los usuarios, se puedan poner en sus zapatos, eso es lo más importante. Tratamos de que siempre ocurra por- que sabemos que, si no se da, el proyecto no va a funcionar porque terminan haciendo cosas que la gente no quiere o que no necesita. Conocer el verdadero problema (Institución 4, profesor 265).

c) Trabajo localizado

Se trata de que los alumnos lleven a cabo actividades (bajo la coordinación o supervisión del docente) en escenarios naturales que exigen el conocimiento minucioso de estos lugares. Además del diálogo y la reflexión, resulta importante llevar a cabo actividades que impliquen la participación activa de los alumnos. Requiere que sean responsables de su proceso y de ser parte de un colectivo:

Pues es que son prácticas, tiene que haber prácticas no es nada más definir lo que es, hay que entenderlo como práctico si la práctica no la aterrizas, el concepto está padre, el concepto general del ISO te habla de, hablando de responsabilidad social empresarial, te habla de cuidado ecológico, te habla de cuidado a los grupos de interés, cuidado al medio ambiente, o cuidado a la política, como muchas cosas que vemos, está bonita, pero no es funcional, a veces en términos funcionales no es lo más adecuado (Institución 1, profesor 287).

\section{d) Experiencia vicaria}

Estas vivencias cercano-significativas exigen "ponerse en los zapatos" de otros y requieren que los alumnos se impliquen para reconocer profundamente la experiencia de alguien diferente. No sólo se trata de conocer sus condiciones, características y prácticas, sino afrontar las vivencias cotidianas de la otra persona. Este tipo de prácticas son una actividad extraaula común planteada por los docentes.

Hicieron una simulación de un vagón de tren, como en el que se suben los migrantes. Era así en un salón chiquito, todo estaba oscuro y hacía calor, pues como son en realidad los vagones de carga. Y bueno, yo llevé a un grupo de mis chicos, y pues éramos varios y estábamos en el vagón, todos asfixiados, apretados, teníamos calor;

46 - Prácticas docentes que promueven el compromiso social de los alumnos Juan Carlos Silas Casillas, Laura Angélica Bárcenas Pozos. DIDAC 78 (2021): 41-48 
y al salir del salón, en el pasillo había varias fotos de migrantes que tenían una descripción: su nombre, edad, una pequeña historia que ellos habían contado. (Institución 5, profesor 75).

Se emplean frecuentemente videos o películas, así como la visita a lugares en donde se vivan estas realidades diferentes.

No es una clase de apreciación de cine, no me interesa quién es el director, los actores, me interesa que veamos la película desde el contexto de la asignatura y por eso son estas preguntas. Hablando de derecho a la información, cuáles son los problemas jurídicos, sociales que se plantean en el filme, cuál es el problema jurídico-social principal, no siempre es un filme, a veces es un debate o una mesa redonda, con qué argumentos, etcétera. (Institución 4, profesor 140).

Las intenciones que sustentan estas prácticas de acercamiento son tres:

1) Sensibilizar fuertemente al estudiante; ir más allá del intercambio discursivo para que viva de manera vicaria las condiciones y las circunstancias que conoció a través de la conversación.

2) Reconocer las desigualdades sociales en el entorno que las sufre. Se busca que los alumnos asuman cómo se viven las inequidades y reciban la información y la vivencia de los afectados.

3) Convivir directamente con las condiciones de desigualdad de un modo propositivo, reflexivo y controlado.

Estas tres intenciones se logran a través del contacto directo, el aprendizaje situado, el trabajo localizado y la vivencia vicaria. Esto amplía las condiciones para el desarrollo del compromiso social con quienes tienen pocas posibilidades de salir de esta situación. Los estudiantes aprehenden elementos de la realidad social y comprenden cómo aplicar los conocimientos y las habilidades de su carrera en un contexto específico, marcado por la desigualdad.

\section{Conclusiones}

Los resultados son profundamente significativos, ya que los estudiantes toman conciencia sobre otras realidades y actúan en ellas para mejorarlas. Este tipo de prácticas vivenciales, a la par del diálogo y la discusión analítica, permiten experiencias que marcan la vida de los jóvenes y cimientan su compromiso con la equidad social.

Resulta importante señalar que estas prácticas se vuelven educativas y no sólo docentes, pues las propias instituciones jesuitas propician, gestionan y favorecen este tipo de prácticas docentes, tal como lo define García Cabrero:

el conjunto de situaciones enmarcadas en el contexto institucional y que influyen en los procesos de enseñanza y aprendizaje propiamente dichos; se refiere a cuestiones más allá de las interacciones entre profesores y alumnos en el salón de clases, determinadas en gran medida por las lógicas de gestión y organización institucional del centro educativo. (2008, pp. 3-4).

Sin embargo, en estos tiempos de violencia e inseguridad, es muy difícil obtener un resultado completo en todos los casos; tampoco es sencillo que la perdurabilidad experiencial resista el paso del tiempo. A su vez, las presiones sociales obligan a los estudiantes a volver rápidamente a sus costumbres y rutinas. Aun así, es posible dejar una marca en el criterio de los jóvenes, al menos durante sus años formativos.

\section{Recomendaciones para el aula}

- Reconocer las posibilidades de impactar realmente en la formación de los alumnos.

- Proponer diálogos constantes en clase, en los que se manejen temas sociales y cómo los jóvenes pueden ayudar a mejorar las condiciones.

- En la medida de lo posible, llevar a los alumnos a las realidades desiguales, con el propósito de generar un impacto más contundente en el fomento al compromiso social. 
Aguirre, R., Pelekais, C. de \& Paz, A. (2012). Responsabilidad social: compromiso u obligación universitaria. Telos, 14(1), 11-20. Recuperado de https://www.uv.mx/APPS/CUO/ TALLERRSU/Docs.\%20RSU\%20para\%20INEGI/ Responsabilidad\%20social\%20compromiso.pdf

Cacho Sánchez, Y. \& Llano Martínez, L. (2014). ¿Es posible una política universitaria en materia de compromiso social? La experiencia del grupo de compromiso social de la Universidad de Cantabria. Revista Interuniversitaria de Formación del Profesorado, 28(2), 79-92. Recuperado de https://www.redalyc.org/articulo.oa?id=27433840008

Coll, C. \& Solé, I. (2002). Enseñar y aprender en el contexto del aula. En. C. Coll, J. Palacios \& A. Marchesi (Comps.), Desarrollo psicológico y educación 2. Psicología de la educación escolar (pp. 357-386). Madrid: Alianza Editorial.

García Cabrero, B., Loredo Enríquez, J. \& Carranza Peńa, G. (2008). Análisis de la práctica educativa de los docentes: pensamiento, interacción y reflexión. Revista Electrónica de Investigación Educativa, 10. Recuperado de http://www.scielo.org.mx/scielo.php?script=sci_arttext $\&$ pid=S1607-40412008000300006

López Zavala, R. (2007). Valores profesionales en la formación universitaria. La dimensión social de los valores del profesorado. REencuentro. Análisis de Problemas Universitarios, 49, 59-64. Recuperado de https://www.redalyc.org/articulo.oa?id=34004909

Martínez-Otero, V. (2008). El discurso educativo. Madrid: CCS. Porta, L. G. \& Flores, G. N. (2017). La hospitalidad en profesores memorables universitarios. REXE. Revista de Estudios y Experiencias en Educación, 16(30), 15-31. Recuperado de https://www.redalyc.org/articulo.oa?id=243150283002

Suescun Guerrero, W. D. (2015). El discurso educativo en profesores universitarios bajo la percepción de sus estudiantes. Lengua y Habla, 19, 267-296. Recuperado de https://www.redalyc.org/pdf/5119/511951375018.pdf

Torrego Egido, L. \& Monjas Aguado, R. (2007). Universidad y compromiso social una experiencia de solidaridad en el campus de Segovia. Revista Pedagógica, 20, 69-84.

\section{SEmblanZas}

Juan Carlos Silas Casillas. Doctor en Educational Policy and Leadership por la Universidad de Kansas; maestro en Educación y licenciado en Psicología Educativa por la Universidad del Valle de Atemajac en Guadalajara. De 2004 a 2008 se desempeñó como director del Centro Interdisciplinario de Estudios sobre Calidad de la Educación y Superación de la Pobreza (CIECESP) en la Universidad de Monterrey. De 2008 a la fecha es investigador y docente en el Departamento de Psicología, Educación y Salud del iteso, Universidad Jesuita de Guadalajara. Desde 2016 es el coordinador del Doctorado Interinstitucional en Educación. Es miembro del Sistema Nacional de Investigadores (SNI) nivel I. Sus temas de investigación y publicación se enfocan en el balance público-privado en la educación superior, la relación entre las instituciones educativas y la comunidad, así como el pensamiento científico-crítico en alumnos universitarios.

Laura Angélica Bárcenas Pozos. Doctora en Educación, maestra en Educación Superior y profesora de educación media básica en la especialidad de Matemáticas. Es académica de tiempo completo de la Universidad Iberoamericana Puebla desde 1997 con la categoría de Titular 1, donde ha coordinado el área de Orientación Educativa y de Formación de

Profesores, además de haber sido directora del Centro de Procesos Educativos y coordinadora del Doctorado Interinstitucional en Educación, así como la representante del Campo Estratégico de Acción en Modelos y Políticas Educativas (CEAMOPE). Actualmente es profesora de tiempo de la Dirección de Investigación y Posgrado. Sus temas de investigación son currículum y modelos educativos, y trabaja la línea de sujetos y modelos educativos. Los temas sociales urbanos son su preocupación permanente.

48 - Prácticas docentes que promueven el compromiso social de los alumnos Juan Carlos Silas Casillas, Laura Angélica Bárcenas Pozos. DIDAC 78 (2021): 41-48 